Proceedings of the 4th Polish Symposium on Econo- and Sociophysics, Rzeszów, Poland, May 7-9, 2009

\title{
Share Price Evolution as Stationary, Dependent Continuous-Time Random Walk
}

\begin{abstract}
T. GubieC* And R. Kutner
Division of Physics Education, Institute of Experimental Physics, Faculty of Physics, University of Warsaw Hoża 69, 00-681 Warszawa, Poland

Simple model of share price evolution, which is an extension of Kehr-Kutner-Binder one and MonteroMasoliver models, is presented. The market empirical data inspired the assumptions of the model. The model seems to be the reference one for the study of the short-range correlations in financial data as it considers the observed correlation over two successive jumps of the financial ant.
\end{abstract}

PACS numbers: 02.50.Ey, 02.50.Ga, 05.40.Fb, 02.30.Mv

\section{Introduction}

Since the time when Continuous-Time Random Walk (CTRW) was introduced by Montroll and Weiss [1] and Scher and Lax [2], the list of its applications, e.g., transport in disordered media, electron tunneling, modeling of hydrological problems as well as earthquake, is still growing up. Recently, its application to description of financial time-series was proposed [3-12]. In the traditional CTRW, times duration and jumps are independent random variables and in the most of cases their distribution are static. In financial time-series, such as single share price, strong correlations between successive jumps have been observed [13], i.e., the independence of successive jumps is violated. In the literature [14-16] the CTRW assuming the successive jumps dependence was analyzed, where particles jumping on a lattice strongly favorize backward jumps instead of forward ones. Montero and Masoliver [17] proposed analogous simple solvable model of the CTRW in the context of financial data. In this paper we extended the Montero and Masoliver model to cover both stationary and non-stationary systems.

\section{Empirical basis of our model}

Strong correlations between relative directions of two successive jumps of the stock price was already discussed in the literature [13]. After increase of the price, its successive decrease in the next transaction is about three times more probable than its successive increase, and vice versa. We analyzed tick by tick $\mathrm{KGHM}^{* *}$ stock data since 2000-11-17 till 2009-02-11 [18] and we found (after removing transactions without price changes) that probabilities of positive and negative backward jumps of price equals about 0.76 (with negligible small statistical error). Our observations confirm the result presented in [13], which was based on empirical data collected more

\footnotetext{
* corresponding author; e-mail: Tomasz.Gubiec@fuw.edu.pl

** KGHM - KGHM Polska Miedź S.A., the mining and metallurgy company based in Poland in Lubin. It is one of the biggest companies at Warsaw Stock Exchange.
}

than one decade ago. Right now, it is clear that empirical observation constitutes basis for theoretical modeling. Moreover we checked if other correlations are so important as previously mentioned ones. In Fig. 1 we presented histograms that shows strong correlations in the former case and negligible ones in latter cases.

\section{Solution of the model}

The single trajectory of the financial ant, realized within our CTRW, is a stair-like curve, which consists of waits and jumps. To define the underlying process $\left(t_{1}, r_{1} ; t_{2}, r_{2} ; \ldots ; t_{n}, r_{n}\right)$, where $t_{j}$ means the waiting-time and $r_{j}$ is the jump of the price $(j=1,2, \ldots, n)$, the following conditional probability should be introduced. Namely,

- $\rho\left(r_{n}, t_{n} \mid r_{n-1}, t_{n-1} ; r_{n-2}, t_{n-2} ; \ldots ; r_{2}, t_{2} ; r_{1}, t_{1}\right)$ is the probability that the jump of the price, $r_{n}$, occurred exactly at the end of the waiting time $t_{n}$ conditioned on the trajectory $\left(t_{1}, r_{1} ; t_{2}, r_{2} ; \ldots ; t_{n-1}, r_{n-1}\right)$.

We postulate that the following approximation will be appropriate for analyzed case,

$$
\begin{aligned}
& \rho\left(r_{n}, t_{n} \mid r_{n-1}, t_{n-1} ; r_{n-2}, t_{n-2} ; \ldots ; r_{2}, t_{2} ; r_{1}, t_{1}\right) \\
& \quad \approx \rho\left(r_{n}, t_{n} \mid r_{n-1}\right) \approx h\left(r_{n} \mid r_{n-1}\right) \psi\left(t_{n}\right) .
\end{aligned}
$$

Additionally, we assume that our process is stationary. Now we need the propagators

- $Q(X, t \mid \xi)$ - probability that financial ant, which was initially $(t=0)$ at origin $(X=0)$ arriving there by making a jump $\xi$, exactly at time $t$ jumps to position $X$. We call it the 'sharp probability' and consider it only for $t>0$.

- $P(X, t \mid \xi)$ — probability that financial ant, which was initially $(t=0)$ at origin $(X=0)$ arriving there by making a jump $\xi$, at time $t$ is at position $X$. We call it the 'soft probability'. 

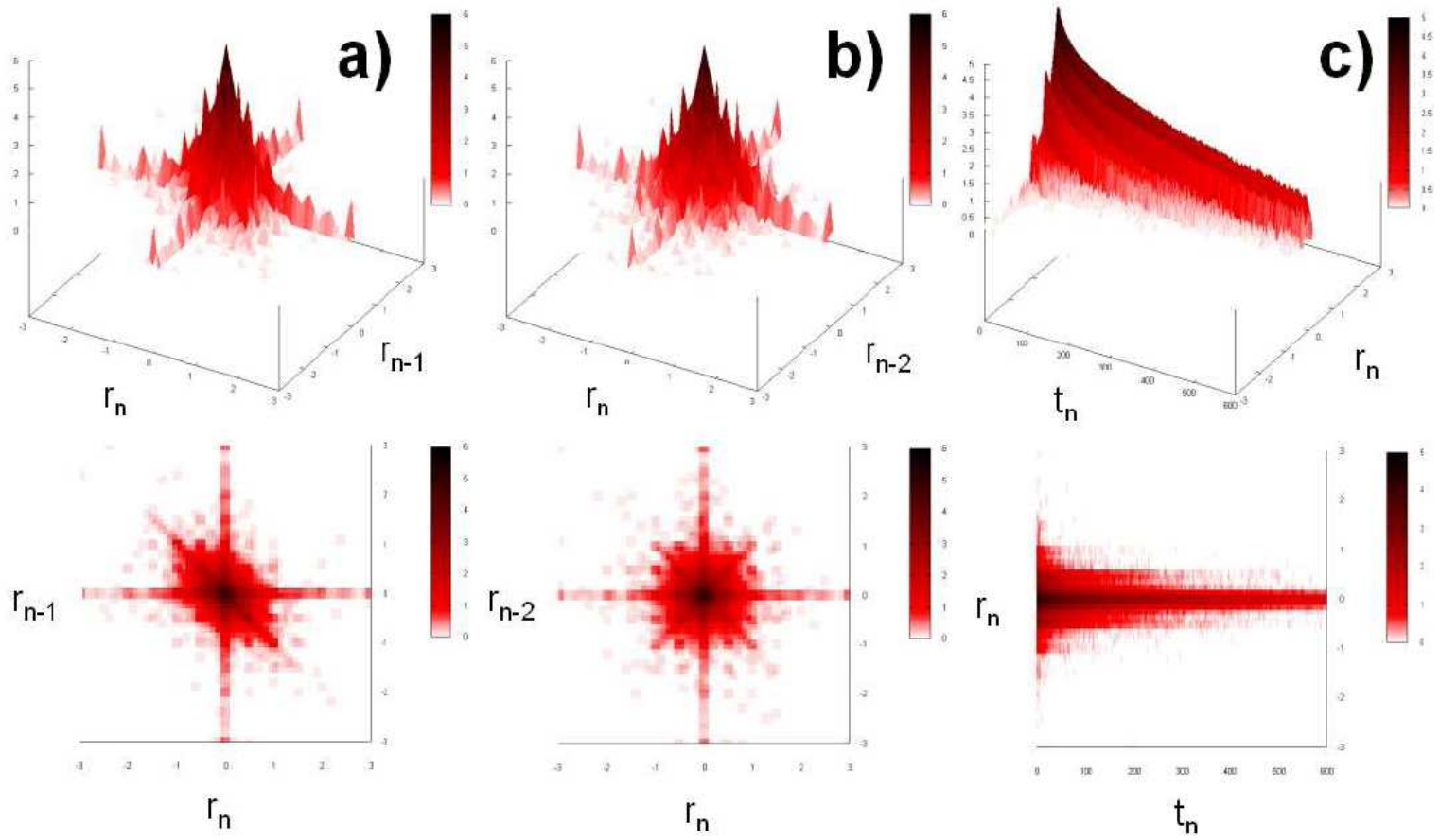

Fig. 1. Analysis of independence between (a) jump $r_{n}$ and previous jump $r_{n-1}$, (b) jump $r_{n}$ and second previous jump $r_{n-2}$, (c) jump $r_{n}$ and time interval between jumps $t_{n}$. $Z$-axis shows a decimal logarithm of the number of counts (increased by one). Jumps lengths are rounded to 0.1 and given in Polish national currency PLN. Time scale is in seconds. Histograms are based on tick by tick KGHM stock data since 2000-11-17 till 2009-02-11 [18]. Histograms (a) shows important correlation between quantities, correlations on histograms (b) and (c) can be neglected in our theoretical approach.

The difference between sharp and soft probabilities is that for the soft one, the ant can arrive to the place earlier and stay there (without jump) till time $t$. In [17] the above mentioned quantities have similar definitions thought small but essential difference exist as there the financial ant arrives to $X=0$ exactly at time $t=0$. This makes $P(X, t \mid \xi)$ non-stationary, in opposite to our stationary case.

The solution of the problem becomes much easier if we use Laplace and Fourier transforms

$$
\tilde{P}(k, s \mid \xi)=\int_{0}^{\infty} \mathrm{d} t \mathrm{e}^{-s t} \int_{-\infty}^{\infty} \mathrm{d} x \mathrm{e}^{\mathrm{i} k x} P(x, t \mid \xi)
$$

analogically we obtain the $\tilde{Q}(k, s \mid \xi)$ quantity. In the approach we decompose

$$
Q(X, t \mid \xi)=\sum_{n=1}^{\infty} Q_{n}(X, t \mid \xi)
$$

where $Q_{n}(X, t \mid \xi)$ is the analogous quantity as $Q(X, t \mid \xi)$ possessing the additional condition that during time $t$ exactly $n$ jumps were made. The component $Q_{0}$ doesn't appear in summarized quantity $Q$ as we consider only $t>0$. Following [17] we assume the conditional jump distribution in the form.

$$
h\left(r_{n} \mid r_{n-1}\right)=h\left(r_{n}\right)\left(1+\epsilon \operatorname{sgn}\left(r_{n}\right) \operatorname{sgn}\left(r_{n-1}\right)\right),
$$

where $h\left(r_{n}\right)$ is unconditional distribution of jumps, which is assumed to be symmetric $h\left(r_{n}\right)=h\left(-r_{n}\right)$.

Now let us separate $\tilde{Q}_{n}(k, s \mid \xi)$ into two parts: $\tilde{Q}_{n}^{+}(k, s \mid \xi)$ - obeying an additional condition that the last jump was positive, and analogously $\tilde{Q}_{n}^{-}(k, s \mid \xi)$, where the last jump was negative. For simpler notation we define half-Fourier transformation

$$
\tilde{H}(k)=\int_{0}^{\infty} \mathrm{d} r \mathrm{e}^{\mathrm{i} k r} h(r), \quad \tilde{h}(k)=\tilde{H}(k)+\tilde{H}(-k) .
$$

We can write recurrent equations for $\tilde{Q}_{n}^{+}, \tilde{Q}_{n}^{-}$, respectively expressed in the matrix form (for $n \geq 2$ )

$$
\begin{aligned}
& \left(\begin{array}{c}
\tilde{Q}_{n}^{+}(k, s \mid \xi) \\
\tilde{Q}_{n}^{-}(k, s \mid \xi)
\end{array}\right)=\tilde{\psi}(s)\left(\begin{array}{cc}
\tilde{H}(k)(1+\epsilon) & \tilde{H}(k)(1-\epsilon) \\
\tilde{H}(-k)(1-\epsilon) & \tilde{H}(-k)(1+\epsilon)
\end{array}\right) \\
& \quad \times\left(\begin{array}{c}
\tilde{Q}_{n-1}^{+}(k, s \mid \xi) \\
\tilde{Q}_{n-1}^{-}(k, s \mid \xi)
\end{array}\right) .
\end{aligned}
$$

Now, let us summarize both sides of Eq. (4) over $n$ and add the postulated form of $\tilde{Q}_{1}^{ \pm}(k, s \mid \xi)$

$$
\begin{gathered}
\left(\begin{array}{c}
\tilde{Q}^{+}(k, s \mid \xi) \\
\tilde{Q}^{-}(k, s \mid \xi)
\end{array}\right)=\tilde{\psi}(s)\left(\begin{array}{cc}
\tilde{H}(k)(1+\epsilon) & \tilde{H}(k)(1-\epsilon) \\
\tilde{H}(-k)(1-\epsilon) & \tilde{H}(-k)(1+\epsilon)
\end{array}\right) \\
\quad \times\left(\begin{array}{c}
\tilde{Q}^{+}(k, s \mid \xi) \\
\tilde{Q}^{-}(k, s \mid \xi)
\end{array}\right)+\tilde{\psi}_{1}(s)\left(\begin{array}{c}
\tilde{H}(k) \\
\tilde{H}(-k)
\end{array}\right) .
\end{gathered}
$$


Note that, for the first jump we cannot use the same waiting time distribution as for other ones, because previous jump might have occurred at any time before $t=0$. In such case we have to average over all possible times of the previous jump. Following [13], we assume

$$
\psi_{1}(t)=\frac{\int_{0}^{\infty} \mathrm{d} t^{\prime} \psi\left(t+t^{\prime}\right)}{\int_{0}^{\infty} \mathrm{d} t^{\prime \prime} \int_{0}^{\infty} \mathrm{d} t^{\prime} \psi\left(t^{\prime \prime}+t^{\prime}\right)} .
$$

The only case when $\psi_{1}(t)=\psi(t)$ is an exponential distribution. The Laplace transform $\tilde{\psi}_{1}(s)$ of $(6)$ can be expressed by the transform of the original waiting time distribution

$$
\tilde{\psi}_{1}(s)=\frac{1-\tilde{\psi}(s)}{\langle t\rangle s},
$$

where $\langle t\rangle=\int_{0}^{\infty} t \psi(t) \mathrm{d} t$. The final form of the stationary propagator, including averaging over sign of $\xi$, is given by

$$
\begin{aligned}
& \tilde{P}(k, s)=\tilde{\Psi}(s)\left(\tilde{Q}^{+}(k, s \mid \xi)+\tilde{Q}^{-}(k, s \mid \xi)\right)+\tilde{\Psi}_{1}(s) \\
& \quad=\left[1-\epsilon \tilde{h}(k) \tilde{\psi}(s)+\left(\tilde{\psi}_{1}(s)-\tilde{\psi}(s)\right)\right. \\
& \quad \times(\tilde{h}(k)-4 \epsilon \tilde{H}(k) \tilde{H}(-k) \tilde{\psi}(s))] \\
& \quad /\left[1-\tilde{h}(k)(1+\epsilon) \tilde{\psi}(s)+4 \epsilon \tilde{H}(k) \tilde{H}(-k) \tilde{\psi}(s)^{2} \tilde{\Psi}(s)\right] \\
& \quad+\left(\tilde{\Psi}_{1}(s)-\tilde{\Psi}(s)\right) .
\end{aligned}
$$

By using this explicit form we can calculate the Laplace transformation of the variance of the process

$$
\begin{gathered}
\tilde{m}_{2}(s)=-\left.\frac{\partial^{2} \tilde{P}(k, s)}{\partial k^{2}}\right|_{k=0}=\frac{\mu_{2} \tilde{\psi}_{1}(s)}{s(1-\tilde{\psi}(s))} \\
+\frac{2 \epsilon \mu_{1}^{2} \tilde{\psi}(s) \tilde{\psi}_{1}(s)}{s(1-\tilde{\psi}(s))(1-\epsilon \tilde{\psi}(s))}
\end{gathered}
$$

where

$$
\mu_{1}=\int_{-\infty}^{\infty} \mathrm{d} x|x| h(x), \quad \mu_{2}=\int_{-\infty}^{\infty} \mathrm{d} x x^{2} h(x) .
$$

Technically, to obtain the result for the non-stationary case it is sufficient to put $\tilde{\psi}(s)$ instead of $\tilde{\psi}_{1}(s)$. Hence, Eqs. (8) and (9) reproduce the corresponding results shown in [17]. We derive variance in the time domain in the form

$$
m_{2}(t)=\frac{\mu_{2}}{\langle t\rangle} t+\frac{2 \epsilon \mu_{1}^{2}}{\langle t\rangle} \mathcal{L}^{-1}\left\{\frac{\tilde{\psi}(s)}{s^{2}(1-\epsilon \tilde{\psi}(s))}\right\}(t),
$$

where $\mathcal{L}^{-1}$ denotes inverse Laplace transform. As we can see, as long as mean time $\langle t\rangle$ exists, there is no matter which is the $\psi(t)$ and $\epsilon$, the asymptotic behavior of the variance is diffusive with diffusion coefficient $D=\mu_{2} /(2\langle t\rangle)$. Additionally, for stationary process we obtain velocity autocorrelation function in the form

$$
C(t)=\frac{\mu_{2}}{2\langle t\rangle} \delta(t)+\frac{\epsilon \mu_{1}^{2}}{\langle t\rangle} \mathcal{L}^{-1}\left\{\frac{\tilde{\psi}(s)}{1-\epsilon \tilde{\psi}(s)}\right\}(t) .
$$

The difference between stationary and non-stationary cases is important for small and intermediate times and it disappears for exponential waiting time distribution $\psi(t)$. To visualize the difference between results in those two cases let us consider waiting time distribution in the form of two-step Poisson process.

$$
\psi(t)=\lambda^{2} t \exp (-\lambda t) .
$$

The difference between stationary variance (obtained from Eq. (11) and its non-stationary analog (shown in [17]), for waiting time distribution given by Eq. (13) takes the form

$$
\begin{aligned}
& \Delta m_{2}(t)=\left\{\mathrm { e } ^ { - \lambda t } \left[\left(2 \epsilon \mu_{1}^{2}-\epsilon \mu_{2}+\mu_{2}\right) \sinh (\lambda t)\right.\right. \\
& \left.\left.-2 \sqrt{\epsilon} \mu_{1}^{2} \sinh (\sqrt{\epsilon} \lambda t)\right]\right\} /[2(1-\epsilon)] .
\end{aligned}
$$

In Fig. 2 two different examples of the difference between stationary and non-stationary variances are plotted for the case of negative (Fig. 2a) and positive (Fig. 2b) $\epsilon$.

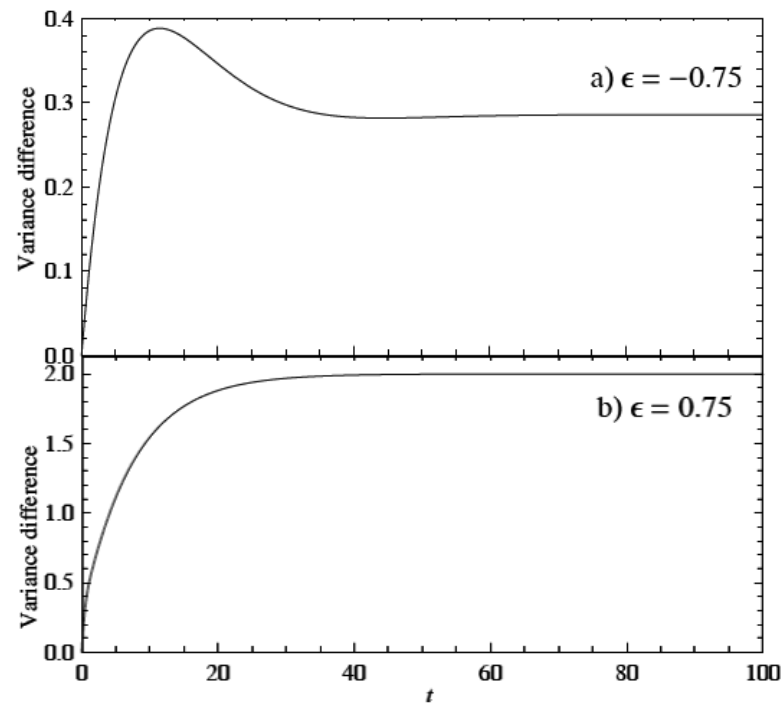

Fig. 2. Difference between stationary and non-stationary variances given by Eq. (14), for $\lambda=1$, $\mu_{1}=1, \mu_{2}=2$. In both cases (negative and positive $\epsilon$ ) difference tends to the constant, what reflects the fact that both variances are asymptotically diffusive, and the difference is important only for small and intermediate times.

\section{Conclusions}

In this work we presented:

- The approach inspired directly by empirical facts, which is important for the study of the short-range correlations, i.e., for short and intermediate times. This was recognized by comparison of the mean-square displacement (variances) for the stationary and non-stationary cases.

- By using Wiener-Khinchine formula (valid only for the stationary situations) we also found the formula 
for the velocity (or price increments) autocorrelation function.

- The achievement of our work is that we found important analytical formulas in a closed form.

\section{Acknowledgments}

We thank Prof. Didier Sornette for very helpful discussions.

\section{References}

[1] E.W. Montroll, G.H. Weiss, J. Math. Phys. 6, 167 (1965).

[2] H. Scher, M. Lax, Phys. Rev. B 7, 4491 (1973).

[3] E. Scalas, R. Gorenflo, F. Mainardi, Physica A 284, 376 (2000).

[4] F. Mainardi, M. Raberto, R. Gorenflo, E. Scalas, Physica A 287, 468 (2000).

[5] M. Raberto, E. Scalas, F. Mainardi, Physica A 314, 749 (2002).

[6] E. Scalas, R. Gorenflo, F. Mainardi, Phys. Rev. E 69, 011107 (2004).
[7] E. Scalas, Physica A 362, 225 (2006).

[8] R. Kutner, F. Switała, Quantitative Finance 3, 201 (2003).

[9] J. Masoliver, M. Montero, G.H. Weiss, Phys. Rev. E 67, 021112 (2003).

[10] P. Repetowicz, P. Richmond, Physica A 344, 108 (2004).

[11] J. Masoliver, M. Montero, J. Perelló, G.H. Weiss, J. Econom. Behav. Organ. 61, 577 (2006).

[12] J. Masoliver, M. Montero, J. Perelló, G.H. Weiss, Physica A 379, 151 (2007).

[13] R.S. Tsay, Analysis of Financial Time Series, Wiley, New Jersey, 2005.

[14] K.W. Kehr, R. Kutner, K. Binder, Phys. Rev. B 23, 4931 (1981)

[15] R. Kutner, J. Phys. C, Solid State Phys. 18, 6323 (1985).

[16] J.W. Haus, K.W. Kehr, Phys. Rep. 150, 263 (1987).

[17] M. Montero, J. Masoliver, Phys. Rev. E 76, 061115 (2007).

[18] http://bossa.pl . 